\title{
Transcranial Direct Current Stimulation Alleviates the Chronic Pain of Osteoarthritis by Modulating NMDA Receptors in Midbrain Periaqueductal Gray in Rats
}

\author{
Xinhe Li, Wenwen Zhou, Lin Wang, Yinshuang Ye, Tieshan Li \\ Department of Rehabilitation Medicine, The Affiliated Hospital of Qingdao University, Qingdao, Shandong Province, People's Republic of China \\ Correspondence: Tieshan Li, Email tieshanl@I26.com
}

\begin{abstract}
Purpose: Osteoarthritis (OA) is the most common cause to lead to chronic pain. Transcranial direct current stimulation (tDCS) has been widely used to treat nerve disorders and chronic pain. The benefits of tDCS for chronic pain are apparent, but its analgesic mechanism is still unclear. This study observed the analgesic effects of tDCS on OA-induced chronic pain and the changes of NMDA receptor levels in PAG after tDCS treatment in rats to explore the analgesic mechanism of tDCS.

Methods: After establishing chronic pain by injecting monosodium iodoacetate (MIA) into the rat ankle joint, the rats received tDCS for 14 consecutive days ( $20 \mathrm{~min} /$ day). Before tDCS treatment, Ifenprodil (the selective antagonist of NMDAR2B) was given to rats in different ways: intracerebroventricular (i.c.v.) injection or intraperitoneal (i.p.) injection. The Von Frey and hot plate tests were applied to assess the pain-related behaviors at different time points. The expression level of NMDAR2B was evaluated in midbrain periaqueductal gray (PAG) by Western blot. In addition, NMDAR2B and c-Fos were observed by the Immunohistochemistry staining after tDCS treatment.
\end{abstract}

Results: The mechanical allodynia and thermal hyperalgesia were produced after MIA injection. However, tDCS treatment reverted the mechanical allodynia and thermal hyperalgesia. Moreover, tDCS treatment significantly increased the expression of NMDAR2B and the proportion of positive stained cells of NMDAR2B. Besides that, the tDCS treatment also decreased the proportion of positive stained cells of c-Fos in PAG. However, these changes did not occur in the rats given the Ifenprodil (i.c.v.).

Conclusion: These results indicate that tDCS may increase the expression of NMDA receptors in PAG and strengthen the NMDA receptors-mediated antinociception to alleviate OA-induced chronic pain in rats.

Keywords: transcranial direct current stimulation, osteoarthritis, chronic pain, NMDA receptors

\section{Introduction}

Osteoarthritis (OA) is a common degenerative joint disease, ${ }^{1}$ and pain is a major clinical problem of OA. ${ }^{2}$ Patients may experience chronic pain ${ }^{3}$ and a decreased quality of life with the development of OA. Traditionally, a combination of pharmacological and nonpharmacological therapies was used to manage the OA pain. ${ }^{4}$ On the one hand, these treatments can reduce the pain intensity; on the other hand, they can also lead to lots of adverse effects, and the treatment benefits may reduce over time. ${ }^{5}$ As the OA pain is often insufficiently treated, many patients suffer joint replacement operation in order to alleviate pain, ${ }^{6}$ but $20 \%$ of patients still have pain after joint replacement therapy. ${ }^{7}$

It is challenging to cure OA pain because the mechanism of chronic pain is complex, involving not only hypersensitivity to pain in the peripheral nervous system (PNS) and the central nervous system (CNS), but also a change in pain modulatory pathways. ${ }^{8}$ Pain modulation pathways may play a vital role in chronic pain. ${ }^{9}$ Persistent and severe pain may decrease the activity of descending inhibitory systems, which results in the persistence of chronic pain. ${ }^{10}$ The periaqueductal gray (PAG)-rostral ventromedial medulla (RVM)-spinal dorsal horn (DH) pathway was known as the key in pain 
modulatory pathways. ${ }^{11}$ The PAG receives the nociceptive information from the spinal cord DH, and it projects antinociceptive transmission to the RVM and lower brainstem, then the RVM projects the information to the spinal cord $\mathrm{DH}^{12-14}$ There are abundant neurotransmitters and receptors in PAG; previous study has shown the effect of excitatory amino acid (EAA) on antinociceptive processing at some PAG sites, the antinociceptive activity of glutamate was dose-dependent and this effect is apparently mediated by N-methyl-D-aspartate (NMDA) receptors. ${ }^{15}$ The NMDA receptors induced activation of the descending inhibitory systems and counteracted the establishment of dorsal horn neural sensitization to produce the antinociception. ${ }^{16,17}$ In four NMDA receptors, the NR2B-containing NMDA receptor played an essential role in pain regulation, ${ }^{18}$ and it was considered one of the best potential target of pain. ${ }^{19-21}$ In addition, the expression of c-Fos is also considered to be one of the most useful parameters for the investigation of the neuronal response to a painful experience. ${ }^{22,23}$

Transcranial direct current stimulation (tDCS) is a non-invasive neuromodulatory technique that modulates neuronal excitability by applying direct current over the scalp using two electrodes. ${ }^{24,25}$ These changes depend on the polarity of the electrode, anodal stimulation enhances excitability, while cathodal stimulation decreased excitability. ${ }^{26}$ A previous study found that tDCS has two effects: the short-term effects are mediated by ionic channel modulation, the long-term effects are mediated by NMDA receptors. ${ }^{27}$ On the one hand, tDCS-induced shifts in the membrane potential can cause lasting excitability changes promoted by the NMDA receptors, and the inward current induced by a particular concentration of glutamate increases with depolarization of the membrane potential. ${ }^{28}$ On the other hand, tDCS leads to an NMDA receptor-mediated augmentation of synaptic strength and induces long-lasting effects by synaptic modification of pain circuits. ${ }^{29,30}$ Transcranial direct current stimulation has been studied in patients with various disorders and multiple pain syndromes. ${ }^{31,32}$ In previous clinical studies of OA pain, it has been proved that M1-SO applied tDCS with a constant direct current of $2 \mathrm{~mA}$ intensity for $20 \mathrm{~min}$ once a day for five consecutive days was efficacious in reducing the OA pain. ${ }^{33,34}$ However, the analgesic mechanism of tDCS is still unclear.

This study aims to investigate whether tDCS can relieve chronic pain caused by OA and explore its mechanism in rats. Once the analgesic mechanism of tDCS in rats is explored clearly, it will be very helpful to generalize it to humans with OA pain.

\section{Materials and Methods}

\section{Animals}

Experiments were implemented on Sprague-Dawley rats $(\mathrm{n}=49$, weight $180 \pm 20 \mathrm{~g})$, which were purchased from the Experimental Animal Center of Qingdao University. These rats were maintained in a controlled environment $\left(22 \pm 2^{\circ} \mathrm{C}\right.$ room temperature and dark/light cycle $12 \mathrm{~h} / 12 \mathrm{~h}$ ) with water and chow ad libitum. There are 5 rats in each cage. The protocol for this experimental study was approved by the Animal Care and Use Committee of Qingdao University and carried out according to the International Association for the Study of Pain. ${ }^{35}$

\section{Experimental Design}

Rats were habituated to the maintenance room for 1 week prior to the experiment. After the habituation period, the rats were divided into seven groups at random: Sham group, MIA group, MIA+tDCS group, MIA+StDCS group, MIA + Ifenprodil (i.c.v.)+tDCS, MIA+PBS (i.c.v.)+tDCS, as well as MIA+Ifenprodil (i.p.)+tDCS. Rats were numbered from 1 to 49 , and the randomization list ( $\mathrm{n}=7$ /group) was established using a computer software (SPSS 26.0). Except for the Sham group, the other six groups received injections of MIA. Before tDCS treatment, two groups of rats were injected (i. c.v.) with Ifenprodil and PBS respectively, and one group was injected with Ifenprodil (i.p.). Then the tDCS and StDCS sessions were applied for 14 days (20 min/day) after 21 days of MIA injection (Figure 1A).

\section{Animal Model}

All rats were light anesthetized with $5 \%$ isoflurane in $\mathrm{O}_{2}$ before inducing OA chronic pain model, and then rats were injected with $60 \mu \mathrm{L} 80 \mathrm{mg} / \mathrm{mL}$ monosodium iodoacetate (MIA, Sigma, USA) into the left ankle joint cavity. ${ }^{36}$ Similarly, saline was injected into the left ankle joint cavity in the Sham group. 
A

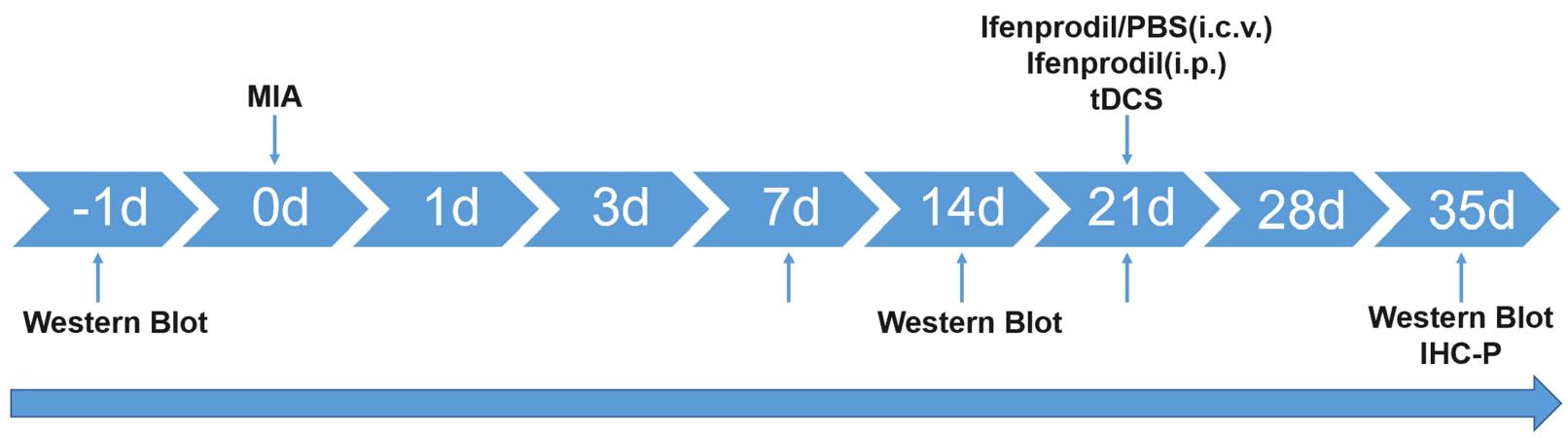

Pain behavioral testing at each time point

B

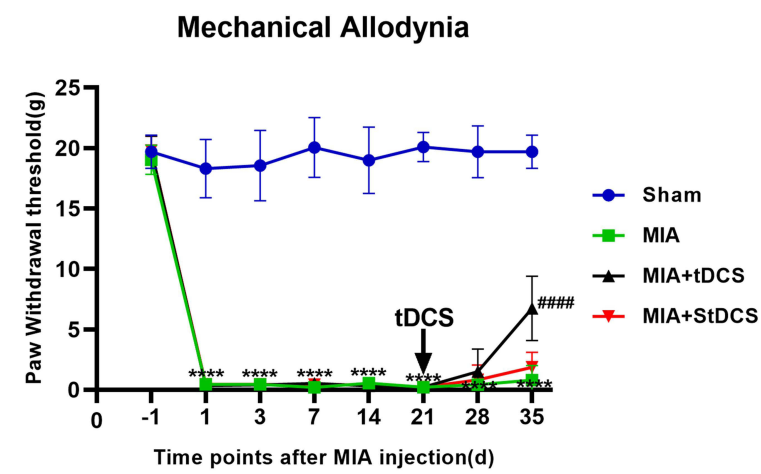

D

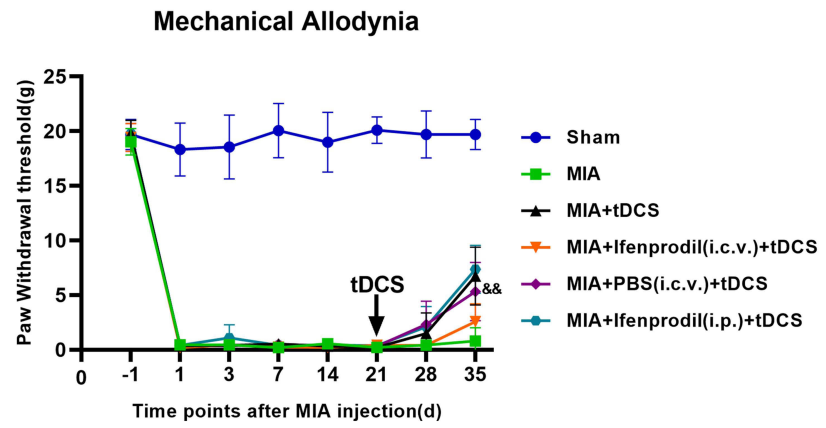

C

Thermal Hyperalgesia

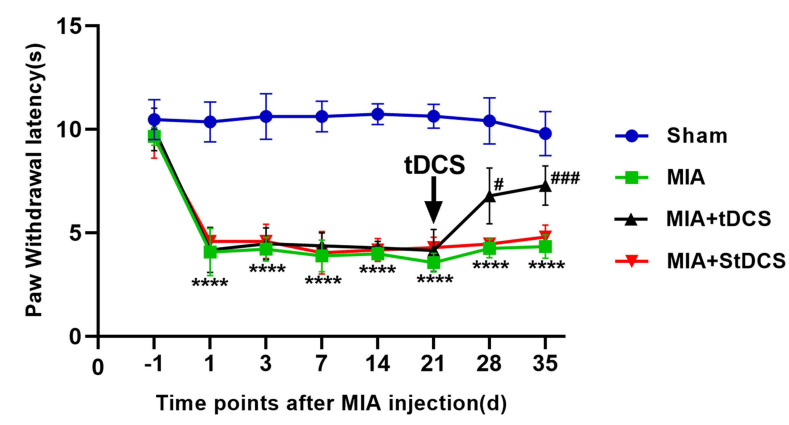

E

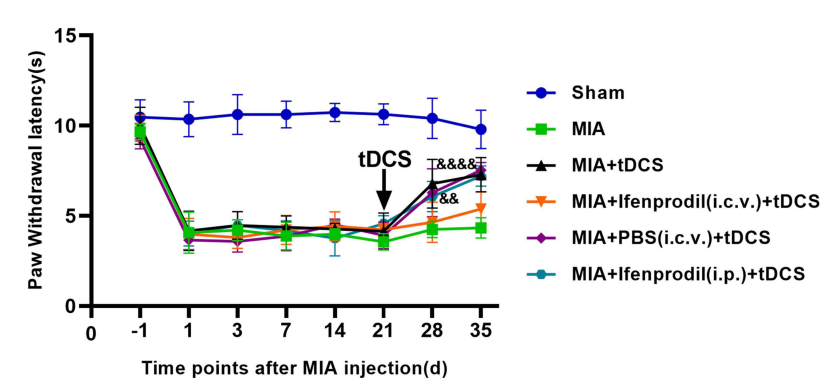

Figure I (A) Experimental design. (B and C) Effects of tDCS on mechanical allodynia (presented by PWT) and thermal hyperalgesia (presented by PWL) in MIA-induced chronic pain rats were shown in the figure. Compared with the Sham group, there were significant decreases on PWT and PWL from I to 35 days after MIA injection. After tDCS treatment, PWT and PWL dramatically increased compared with the MIA group, but no difference was observed between the MIA and MIA+StDCS groups. Data were

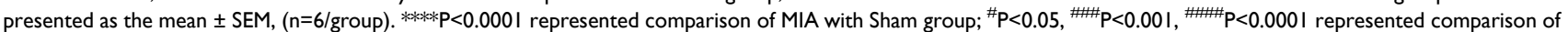
$M I A+t D C S$ with MIA group. (D and E) Effects of Ifenprodil on tDCS in improving mechanical allodynia (presented by PWT) and thermal hyperalgesia (presented by PWL). The PWT and PWL in MIA+PBS (i.c.v.)+tDCS as well as MIA+Ifenprodil (i.p.)+tDCS groups increased significantly, but these changes did not appear in MIA+Ifenprodil (i.c.v.) $+t D C S$ group. There was significant difference in PWT and PWL between the MIA+PBS (i.c.v.) $+\mathrm{tDCS}$ and the MIA+Ifenprodil (i.c.v.) $+\mathrm{tDCS}$ groups. Data were presented as the mean \pm SEM, ( $n=6 /$ group). ${ }^{\& \&} P<0.01$, ${ }^{\& \& \& \&} \mathrm{P}<0.000$ I represented comparison of MIA+PBS (i.c.v.) + tDCS with MIA+lfenprodil (i.c.v.)+tDCS group.

Abbreviations: MIA, Monosodium lodoacetate; PBS, Phosphate Buffered Saline; i.c.v., intracerebroventricular; i.p., intraperitoneal; tDCS, transcranial direct current stimulation; IHC-P, Immunohistochemistry-Paraffin sections.

\section{Stereotaxic Surgery Microinjection Procedures}

The rats were anesthetized with the combination of intraperitoneal $90 \mathrm{mg} / \mathrm{kg}$ ketamine and $10 \mathrm{mg} / \mathrm{kg}$ xylazine. Each rat was placed in the stereotaxic apparatus while maintaining the incisor bar at $3.3 \mathrm{~mm}$ below horizontal to achieve a flat skull position. A 26-gauge guide cannula was targeted at the right midbrain periaqueductal gray (coordinates: midline $= \pm 0.7 \mathrm{~mm}$; anterior posterior $=-7.8 \mathrm{~mm}$; dorsoventral $=-5.6 \mathrm{~mm}$ from Bregma) according to the Paxinos and Watson rat brain atlas. ${ }^{37}$ 
The guide cannula was fixed in position with steel screws and dental cement. Then, a dummy cannula was inserted and fixed with a dust cap. At the end of the surgery, subjects were injected (i.p.) with an antibiotic association to prevent possible infections. Rats were allowed $2 \mathrm{~d}$ to recover from surgery. Drug infusions were done by a 33-gauge stainless steel needle ( $1 \mathrm{~mm}$ longer than the guide cannula) attached to a Hamilton micro-syringe via polyethylene tubing. $1 \mu \mathrm{g}$ of the Ifenprodil (Sigma-Aldrich) was dissolved in $0.1 \mathrm{M}$ phosphate-buffered saline (PBS; $\mathrm{pH} 7.4$ ) at a total volume of $0.3 \mu \mathrm{L}$. Ifenprodil or PBS injection was infused for $8 \mathrm{~min}$, and after the infusion, the syringe was kept at the injection site for 10 more minutes for the absorption of the chemical. The microinjection was performed twice a week until the rats were sacrificed.

\section{Intraperitoneal Injection of Ifenprodil}

The Ifenprodil was diluted in PBS ( $\mathrm{pH}$ 7.4) to prepare for injection. The seven rats in MIA+Ifenprodil (i.p.)+tDCS group were light anesthetized with $5 \%$ isoflurane in $\mathrm{O}_{2}$. I.p. injection $(3 \mathrm{mg} / \mathrm{kg})$ was performed twice a week using a 28 -gauge needle until the rats were sacrificed.

\section{TDCS Protocol}

After 21 days of MIA administration, the rats in six groups received a constant direct current of $0.5 \mathrm{~mA}$ for fourteen consecutive days (20 minutes/day). After the rats were briefly anesthetized by $5 \%$ isoflurane in $\mathrm{O}_{2}$, and then using bandages to bind their limbs to keep the rats immobile. In order to fit the rats' heads, we reduced the size of the electrodes to $1.5 \mathrm{~cm}^{2}$. In addition, the rats' heads were shaved for better adherence before application. ${ }^{38,39}$ Our stimulation parameters provided a current density of $0.33 \mathrm{~mA} / \mathrm{cm}^{2}$ with no lesions for the brain. The cathode electrode was positioned on the midpoint between the lateral angles of both eyes (supraorbital area), and the anode electrode was placed at the head using landmarks of the neck and shoulder lines as a guide (the anterior and posterior regions in the midline between the two hemispheres of the parietal cortex). After the electrodes had been placed, they were fixed onto the head with adhesive tape. Sham-stimulated rats underwent similar procedures, but the stimulator was turned off throughout the experiments. ${ }^{40}$

\section{Behavioral Testing Mechanical Allodynia}

The Von Frey hair (North Coast, USA) based on the up-down method was used to assess mechanical allodynia. ${ }^{41}$ Behavioral test was performed in a blinded manner, and the observer was not clear about the grouping in advance. Before testing, the rats were placed in the cages with a metal mesh floor and acclimatized to the environment for 10 minutes to prevent the analgesia induced by the novelty of the apparatus. Paw withdrawal threshold (PWT) was evaluated in all SD rats at different time points. PWT was assessed in ascending order of force $(0.16 \mathrm{~g}-26.0 \mathrm{~g})$ to the mid-plantar surface vertical to the paw, while the tests invariably began with $2 \mathrm{~g}$, and each hair was applied 5 times at $7 \mathrm{~s}$ intervals. For the mechanical stimulation, the paw's rapid withdrawal or licking was considered to be a positive reaction. If positive responses did not occur more than twice during 5 applications of a particular hair, the next ascending hair would be applied in a similar manner. If we observed positive responses from a particular hair 3 out of the five consecutive applications, the value of a particular hair in that gram was considered to be the withdrawal threshold. According to the up-down method, the $50 \%$ response threshold was interpolated using the formula: $50 \% \mathrm{~g}$ threshold $=\left(10^{[\mathrm{Xf}+\mathrm{K} \&]}\right) / 10,000$. Ultimately, the measurements were averaged in each group.

\section{Thermal Hyperalgesia}

All rats were exposed to a hot plate (HP) for $5 \mathrm{~min}$ to adapt to the hot plate $24 \mathrm{~h}$ before testing. ${ }^{42}$ On the test day, the surface of the plate was kept at a constant temperature $\left(55 \pm 0.1^{\circ} \mathrm{C}\right)$. The rats were placed in glass funnels on the heated surface, and the time in seconds for foot-licking or jumping was recorded as the paw withdrawal latency (PWL). ${ }^{43}$

\section{Western Blot Analysis}

At $24 \mathrm{~h}$ after the last treatment, all groups ( $\mathrm{n}=3$ /group) were killed by decapitation, and the periaqueductal gray tissues were quickly removed and stored at $-80^{\circ} \mathrm{C}$ until use. After adding protease and phosphatase inhibitors, these tissues were 
homogenized in ice-cold lysis buffer and measured protein concentrations with a BCA Protein Assay Kit (Solarbio, China). Proteins $(32 \mu \mathrm{g})$ were separated on a $10 \%$ sodium dodecyl sulfate polyacrylamide gel (Sigma, USA) and transferred to polyvinylidene fluoride (PVDF) membranes (Sigma, USA). The membranes were incubated with 5\% nonfat dry milk in TBST for 2 hours. Then the membranes were incubated with antibodies to NMDAR2B (diluted 1:5000, Abcam) or GAPDH (diluted 1:5000, Abways) overnight at $4^{\circ} \mathrm{C}$. Next, the membranes were incubated with HRP-labeled goat anti-rabbit IgG secondary antibody (diluted 1:5000, Bioss) for 2 hours after washing with TBST. After a final wash in TBST, bands were visualized using an enhanced chemiluminescence ECL reagent (Millipore, USA). Images were analyzed by Image $\mathrm{J}$ software.

\section{Immunohistochemistry Analysis}

The rats in seven groups ( $\mathrm{n}=4$ /group) were deeply anesthetized and then sequentially perfused with saline and $4 \%$ paraformaldehyde ( $\mathrm{pH}$ 7.4). Subsequently, brains were quickly removed and placed in the perfusion fixative $\left(4^{\circ} \mathrm{C}\right)$ for 24 h. Paraffin-embedded sections of PAG were cut at a $5 \mu \mathrm{m}$ thickness and treated with $0.3 \%$ Triton X-100 and $3 \% \mathrm{H}_{2} \mathrm{O}_{2}$ in PBS for $1 \mathrm{~h}$, and processed for $2 \mathrm{~h}$ in 5\% normal goat serum, then stained with anti-NR2B and anti-c-Fos overnight at room temperature. On the second day, they were reacted with secondary antibodies (diluted 1:1000, Abcam) for $1 \mathrm{~h}$ after washing with PBS. Next, adding the DAB color developing solution until the brown-yellow color appeared, and these sections were redyed with hematoxylin after flushing with running water for 30 minutes, then dehydrated through a series of ethanol solutions, cleared in xylene. Finally, images were obtained on a confocal Olympus Fluoview IX73 microscope.

\section{Statistical Analysis}

All data were expressed as mean \pm S.E.M. in this present study and analyzed by GraphPad Prism 8.0 software (GraphPad Software, CA, USA). The pain thresholds were evaluated by two-way repeated measures analysis of variance (RMANOVA). One-way ANOVA was used for analyzing the differences between the groups for the Western blot and immunohistochemistry staining. In all cases, $p$-values $<0.05$ was considered statistically significant.

\section{Results}

\section{Effects of MIA and tDCS on Mechanical Allodynia and Thermal Hyperalgesia}

No significant differences were observed in PWT and PWL among all groups before MIA injection. Compared with the Sham group, the MIA-induced OA rats showed prolonged allodynia and significantly decreased in PWT (Figure 1B) and PWL (Figure 1C) over the whole process of the measure. Twenty-one days after MIA injection, the chronic pain rats received tDCS or StDCS treatment. The results of analysis showed that there was a partial increase in PWT to mechanical stimulation on 14 days after tDCS treatment compared with the MIA group. Similarly, compared with the MIA group, the PWL was obviously reversed by tDCS treatment. In contrast, there was no significant difference in PWT and PWL between the MIA+StDCS and the MIA groups (Figure 1B and C).

\section{Effects of Ifenprodil on tDCS in Improving Mechanical Allodynia and Thermal Hyperalgesia}

The analysis of behavioral testing showed that there was significant difference in PWT and PWL between the MIA+PBS (i.c.v.) + tDCS and the MIA+Ifenprodil (i.c.v.) + tDCS groups (Figure 1D and E). The PWT and PWL in MIA+PBS (i.c.v.) + tDCS as well as MIA+Ifenprodil (i.p.)+tDCS groups increased significantly, but these changes did not appear in MIA + Ifenprodil (i.c.v.)+tDCS group. These results implied the tDCS treatment did not improve the pain-related behaviors in rats injected (i.c.v.) with Ifenprodil.

\section{TDCS Treatment Increased Expression for NMDAR2B in the PAG}

Twenty-one days after the MIA injection, the Western blot results indicated that there was an intense increase in NMDAR2B level on days 7, 14, and 21 after MIA injection (Figure 2A). In addition, tDCS treatment significantly increased the expression of NMDAR2B in the PAG compared with that in MIA-exposed rats (Figure 2B). Moreover, 


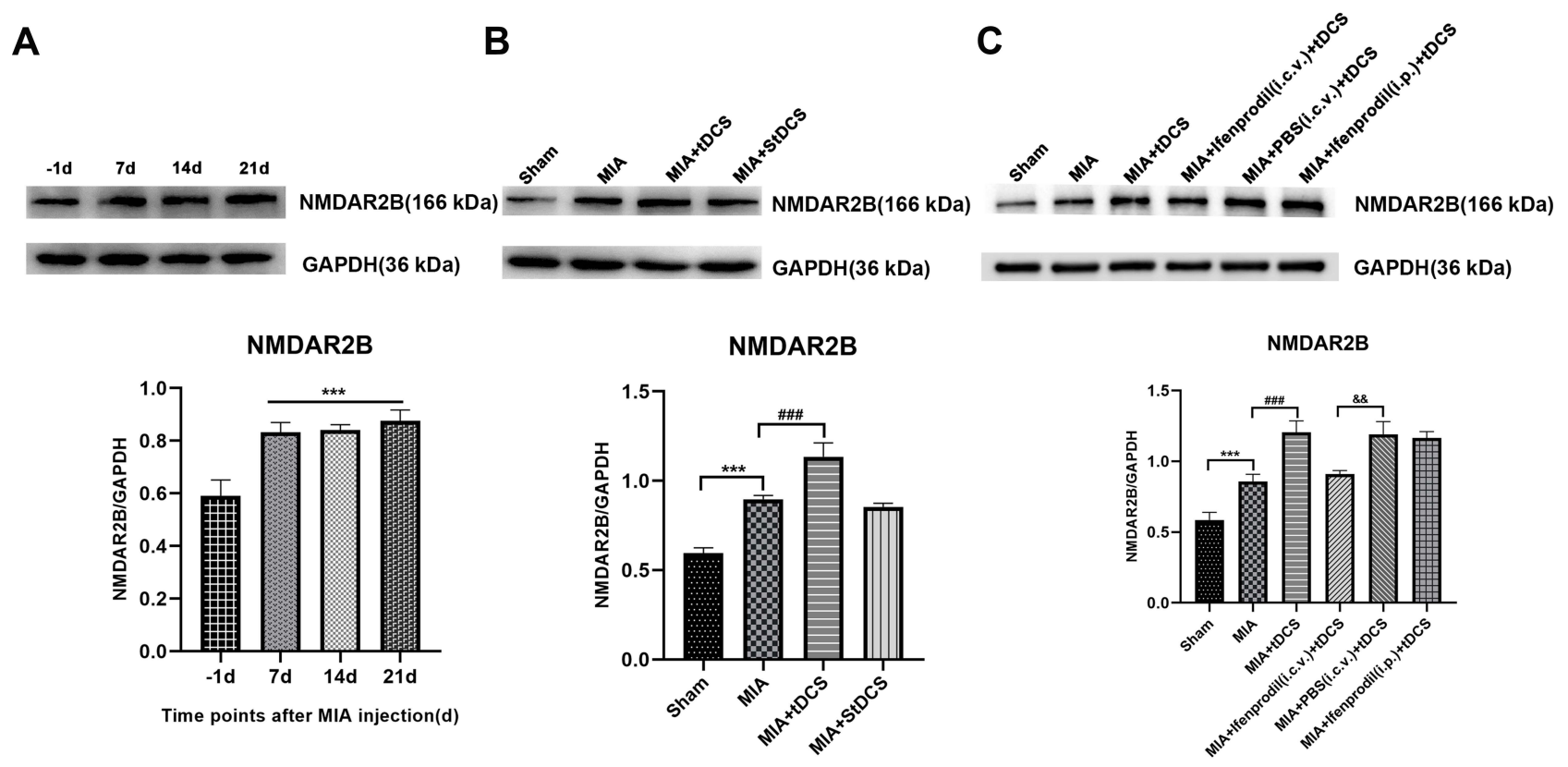

Figure 2 (A) Effect of MIA on NMDAR2B protein in the PAG by Western blot analysis. Data were presented as the mean \pm SEM ( $n=3 / g r o u p)$. $* * * P<0.00 I$, represented comparison of 7 days, 14 days and 21 days after MIA injection with I day before MIA injection. (B) The expression of NMDAR2B protein in the PAG was measured at 14 days after tDCS treatment. Data were presented as the mean \pm SEM ( $n=3 /$ group). ${ }^{* * *} \mathrm{P}<0.00 \mathrm{I}$, MIA group vs Sham group; ${ }^{\prime} \mathrm{P}<0.00 \mathrm{I}$, MIA+tDCS group vs MIA group. (C) The expression of NMDAR2B protein in the PAG was measured at 14 days after $t D C S$ treatment. Data were presented as the mean \pm SEM ( $n=3 / g r o u p$ ). $* * * P<0.00 I$, MIA group vs Sham group; ${ }^{\# \#+0.00 I, ~ M I A+t D C S}$ group vs MIA group; ${ }^{\& \&} \mathrm{P}<0.01$, MIA+PBS (i.c.v.) $+\mathrm{tDCS}$ vs MIA+lfenprodil (i.c.v.)+tDCS group.

there was no significant difference between the MIA+StDCS and the MIA groups. Figure $2 \mathrm{C}$ shows that the tDCS treatment did not increase the expression of NMDAR2B in rats injected with Ifenprodil (i.c.v.). But the expression of NMDAR2B in rats injected with PBS (i.c.v.) and Ifenprodil (i.p.) still increased markedly after the tDCS treatment. Immunohistochemistry also indicated that the proportion of positive stained cells increased in the tDCS treatment group. Compared with the MIA group, tDCS treatment significantly increased the expression of NMDAR2B, while there was no significant difference among MIA and MIA+StDCS groups. Similarly, the proportion of positive stained cells increased in rats injected with PBS (i.c.v.) and Ifenprodil (i.p.), but this change did not occur in MIA+Ifenprodil (i.c.v.) + tDCS group (Figure 3).

\section{TDCS Treatment Decreased Expression for c-Fos in the PAG}

The results of c-Fos staining declared that compared with MIA group, the tDCS treatment reduced the expression of c-Fos in PAG, but there was no decline in c-Fos expression in the rats injected with Ifenprodil (i.c.v.) after tDCS treatment. Moreover, compared with the MIA+Ifenprodil (i.c.v.)+tDCS group, the expression of c-Fos decreased significantly in MIA+PBS (i.c.v.) $+\mathrm{tDCS}$ as well as MIA+Ifenprodil (i.p.)+tDCS groups. And the difference between the MIA and MIA+StDCS groups was not statistically significant (Figure 4).

\section{Discussion}

In the study, we aimed to investigate whether tDCS can relieve OA-induced chronic pain and explore its analgesic mechanism. Our results showed that tDCS reverted mechanical allodynia and thermal hyperalgesia, it also increased the NMDAR2B levels and decreased the expression of c-Fos in PAG at the same time. We concluded that tDCS can modulate the expression of NMDA receptors in PAG and strengthen the NMDA receptors-mediated antinociception to alleviate chronic pain. In order to confirm our inference, the Ifenprodil (the selective antagonist of NMDAR2B) was injected (i.c.v.) into the PAG before the tDCS treatment, the results showed that tDCS treatment did not improve the painrelated behaviors and reverse the NMDAR2B and c-Fos levels. These results hinted that NMDA receptors may be involved in the treatment of chronic pain by tDCS. As we know, the long-term effects of tDCS are mediated by NMDA 
Sham

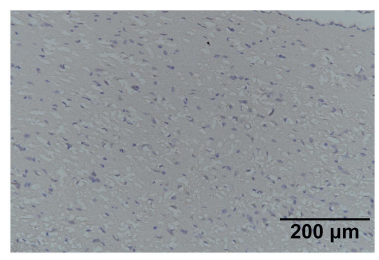

MIA

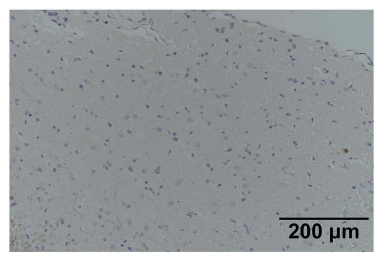

MIA+Ifenprodil(i.c.v.)+tDCS

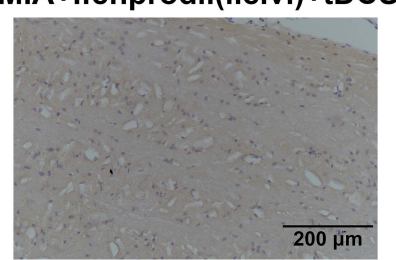

NMDAR2B

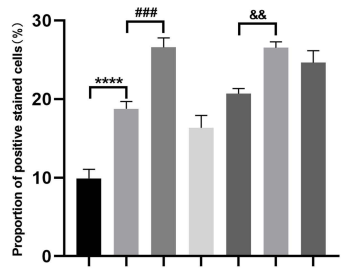

MIA+tDCS

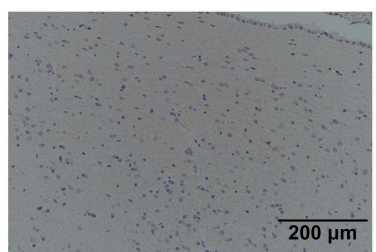

MIA+PBS(i.c.v.)+tDCS

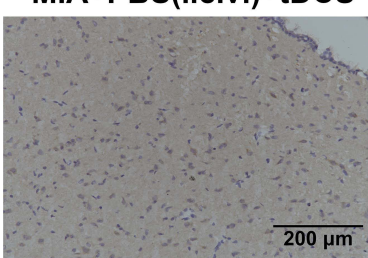

- Sham

- MIA

- MIA+tDCS

MIA+StDCS

- MIA+Ifenprodil(i.c.v.)+tDCS

- MIA+PBS(i.c.v.)+tDCS

- MIA+Ifenprodil(i.p.)+tDCS

\section{MIA+StDCS}

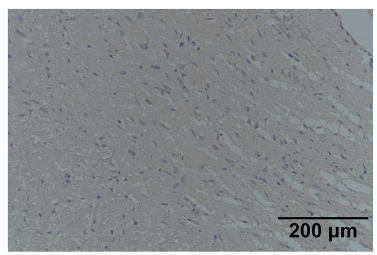

MIA+Ifenprodil(i.p.)+tDCS

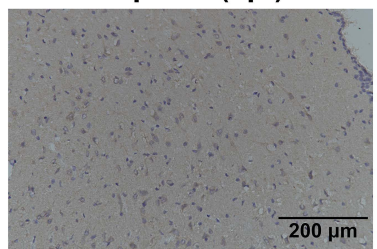

Figure 3 Immunohistochemical staining for NMDAR2B in all groups. Scale bars: $200 \mu \mathrm{m}$. The proportion of positive stained cells were presented as the mean \pm SEM ( $=4$ /

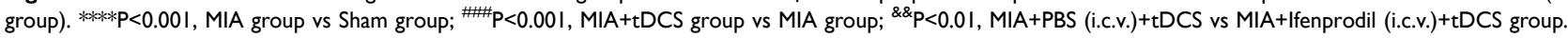

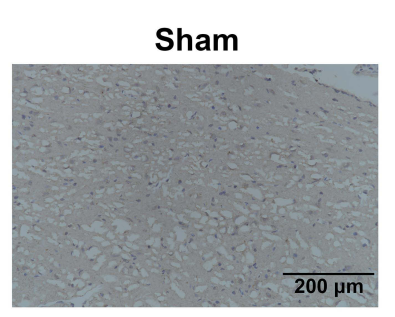

MIA

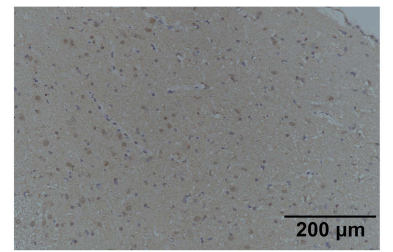

MIA+Ifenprodil(i.c.v.)+tDCS

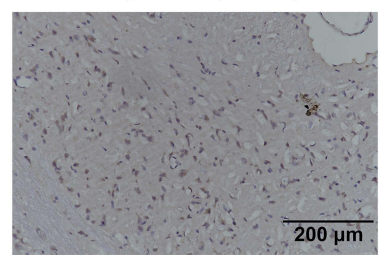

c-Fos

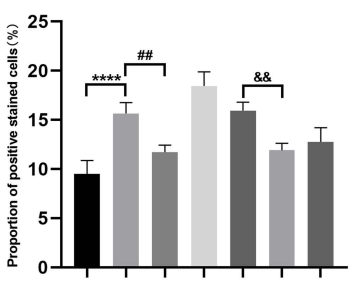

MIA+tDCS

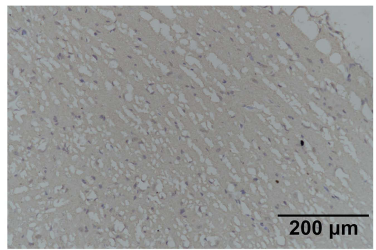

MIA+PBS(i.c.v.)+tDCS

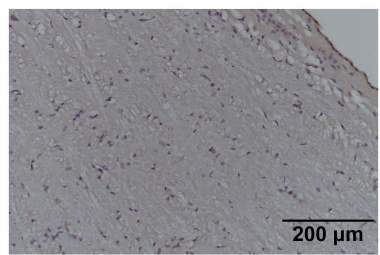

- Sham

- MIA

- MIA+tDCS

MIA+StDCS

- MIA+Ifenprodil(i.c.v.)+tDCS

MIA+PBS(i.c.v.)+tDCS

- MIA+lfenprodil(i.p.)+tDCS

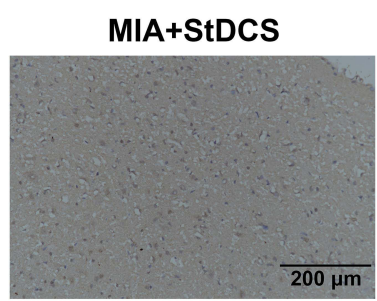

MIA+Ifenprodil(i.p.)+tDCS

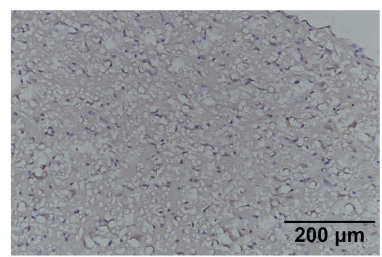

Figure 4 Immunohistochemical staining for c-Fos in all groups. Scale bars: $200 \mu \mathrm{m}$. The proportion of positive stained cells were presented as the mean \pm SEM ( $\mathrm{n}=4 /$ group). $* * * * \mathrm{P}<0.00$ I, MIA group vs Sham group; ${ }^{\# \#} \mathrm{P}<0.0 \mathrm{I}$, MIA+tDCS group vs MIA group; ${ }^{\& \&} \mathrm{P}<0.0 \mathrm{I}, \mathrm{MIA}+\mathrm{PBS}$ (i.c.v.)+tDCS vs MIA+Ifenprodil (i.c.v.)+tDCS group. 
receptors, and no studies explored the changes of NMDA receptor levels in PAG after tDCS treatment so far. Therefore, our research may be an important reference for clarifying the analgesic mechanism of tDCS.

Osteoarthritis (OA) pain is the most frequent cause to lead to chronic pain, and many animal models have been used to study OA chronic pain. A previous study found that a low dose of MIA $(50 \mathrm{mg} / \mathrm{mL})$ failed to induce ongoing pain. ${ }^{44}$ Nevertheless, a high dose of MIA $(80 \mathrm{mg} / \mathrm{mL})$ can not only cause persistent ongoing pain but also may exist a neuropathic pain component in animals, ${ }^{36}$ this study proved that a high dose of MIA injection into the joint cavity could induce chronic pain. In our study, the high dose of MIA was used to establish a chronic pain model; we found the PWT and PWL decreased significantly after MIA injection, and the change of pain behavior lasted for the whole experiment.

In our study, the rats received a 20-min session of tDCS treatment for fourteen consecutive days after 21 days of MIA administration, and we observed that the PWT of the MIA+tDCS, MIA+PBS (i.c.v.)+tDCS as well as MIA+Ifenprodil (i.p.) + tDCS groups gradually increased. Compared with other groups, there was significant difference on the 14th day after the tDCS treatment. Interestingly, the PWL of the above three groups was also significantly different from others on the 7th day after the treatment. The differences of the analgesic response induced by tDCS may be involved in different fibers stimulated by those tests. Thermal nociception is mediated by $\mathrm{C}$ - and $\mathrm{A} \delta$-fibers and mechanical response is mediated by A $\beta$ fibers. ${ }^{45}$ Our results showed that tDCS relieved OA-induced chronic pain, but there may be differences in treatment time for relieving pain caused by different stimuli.

Previous research found that electrical stimulation of the primary motor cortex (M1) can modulate pain, ${ }^{46}$ the effects of tDCS on pain relief depended on the projection of fibers from the motor cortex to other structures involved in pain processing, such as the thalamus and brainstem nuclei that downregulate processing from sensitized neurons, ${ }^{47,48}$ and other researchers found the bimodal tDCS reverted thermal hyperalgesia and partially increased the mechanical threshold in rats subjected to $\mathrm{CCI} .{ }^{40}$ Traditionally, $\mathrm{tDCS}$ was considered to work on the forebrain, such as the primary motor cortex and dorsolateral prefrontal cortex. ${ }^{49}$ However, recent studies found that M1 stimulation can also activate the contiguous regions, including the anterior cingulate cortex, periaqueductal gray (PAG) and amygdala. ${ }^{50,51}$ These researches further confirmed that tDCS could be effective in the direct contact area of the electrode and play a role in the distance. Consistent with previous studies, we also chose M1 as the stimulation site of tDCS.

The midbrain periaqueductal gray (PAG) plays a crucial role in pain modulatory pathways. ${ }^{52}$ It is part of a duplex system of pain control in that it has both nociceptive and antinociceptive effects. ${ }^{53}$ On the one hand, PAG can project the nociceptive input from the spinal cord and medulla oblongata to the thalamus or cerebral cortex to induce nociception; on the other hand, the PAG receives antinociceptive input from the higher center, and it projects this input to the RVM as well as lower brainstem. ${ }^{54}$ The PAG has limited direct projections to the spinal cord, but it uses the RVM as an essential intermediary, then the RVM projects directly to the spinal cord DH. ${ }^{11,12}$ In addition, the PAG is interrelated with all levels of the central nervous system, including diencephalic and forebrain structures related to pain transmission. ${ }^{55}$ These studies further confirmed that PAG is a key site of pain regulation.

In addition to the anatomical structure, we should also consider neurotransmitters and receptors in pain modulatory pathways. There are a great quantity of excitatory amino acids (EAA) and NMDA receptors in PAG. ${ }^{56}$ Previous study found the excitatory amino acids (EAA) are important mediators involved in the adjustment of innate defensive responses produced by PAG. ${ }^{57}$ A point has been proposed by Jensen et al: the antinociceptive activity of glutamate was dose-dependent and the NMDA receptors apparently mediate this effect. In their research, different doses of excitatory amino acid (EAA) agonists were injected into the PAG of rats, and they found that the antinociceptive effect was gradually enhanced with the increase of the dose. At the same time, in order to determine whether EAA antagonists can reverse the antinociceptive effect of EAA agonists, they pretreated rats with different doses of EAA antagonists and then injected EAA agonists into PAG. Interestingly, with the increase of the dose of the antagonist, the antinociceptive effect gradually weakened, and there was a significant decline in tail flick (TF) and hot plate (HP) response latencies. ${ }^{15}$ In addition, Berrino et al suggested that NMDA receptors in PAG may have antinociceptive effects, intra-PAG infusions of NMDA receptors agonists provoked high magnitude antinociception, and this effect was antagonized by the antagonist of NMDA receptors. ${ }^{58}$ Palazzo et al found that physiological NMDA receptor stimulation may be required for cannabinoid-mediated antinociception in the PAG. ${ }^{59}$ In this study, we found the 
expression of NMDAR2B receptor significantly increased after MIA injection, but we did not observe the improvement of pain-related behaviors in rats. We speculated that the increase of NMDAR2B levels in PAG is likely to be a defense response of the body for pain, the amount of the increase did not reach the level that can improve painrelated behaviors in rats. As found by Ramos Coutinho, M, the release of EAA and the activity of NMDA receptors in the PAG can contribute to modulate the duration of the defense response. ${ }^{57}$ Interestingly, we also discovered that NMDAR2B levels increase even more and the PWT and PWL significantly increased after tDCS treatment. We concluded that tDCS treatment may increase more production of EAA and NMDA receptors, and then strengthen antinociceptive effect and the duration of the defense response to revert mechanical allodynia and thermal hyperalgesia. The addition of NMDAR2B antagonists further confirmed our deduction; the injection of Ifenprodil (i.c.v.) made NMDA receptor unable to play the role of analgesia normally after the tDCS treatment. However, the peripheral injection of Ifenprodil (i.p.) did not influence the effect of tDCS, which may be due to the existence of blood-brain barrier (BBB).

C-Fos has been considered as a molecule related to neuronal activation, an increased level of c-Fos expression in supraspinal sites such as the PAG induced by the pretreatment with the CCI operation was demonstrated in the previous study ${ }^{60,61}$ In our study, we found the tDCS treatment decreased the expression of c-Fos, we inferred that the increased activity of NMDA mediated descending inhibitory system may lead to the decreased activity of pain-related neurons, but this still needs further research. It has been proved that the long-lasting effects of tDCS involve the participation of glutamatergic N-methyl-D-aspartic acid (NMDA) receptors, and this role of the glutamatergic system was also demonstrated using D-cycloserine, a partial NMDA agonist, which selectively potentiated the duration of motor cortical excitability induced by anodal tDCS. ${ }^{62}$ Other researchers have found that the changes in NMDA receptors after tDCS might decrease the function of brain areas involved in pain management through long-term potentiation (LTP) synaptic efficacy, thus inducing cortical reorganization and CNS network processing. ${ }^{63,64}$ Compared with previous studies, our research further confirmed the tDCS can activate PAG and produce the antinociceptive effect by regulating NMDA receptors in PAG. Based on the current research results, we can only infer the analgesic mechanism of tDCS in rats and these results may not be applicable to human, further research on human needs to be done in clinical studies.

\section{Conclusion}

Our results demonstrated that tDCS can alleviate OA-induced chronic pain in rats by increasing the expression of NMDA receptors in PAG and strengthening the NMDA receptors-mediated antinociception to play an analgesic role. There are limitations inherent to the current study design and that several questions remain open. More research will be done in the future to explore the mechanism of tDCS.

\section{Ethics Approval}

In this study, the rights and interests of rats were fully protected, which met the requirements of the Laboratory Animal Welfare Ethics Committee of Qingdao University (The approval number: NO.20200901SD4020210301039).

\section{Author Contributions}

TL designed the study. XL executed the study. WZ acquired the data, LW interpreted the data, YY analyzed the data. All authors contributed to data analysis, drafting or revising the article, have agreed on the journal to which the article will be submitted, gave final approval of the version to be published, and agree to be accountable for all aspects of the work.

\section{Funding}

This study was supported by the Natural Science Foundation of Shandong Province, ZR2018MH031.

\section{Disclosure}

The authors report no conflicts of interest. 


\section{References}

1. Poulet B, Staines KA. New developments in osteoarthritis and cartilage biology. Curr Opin Pharmacol. 2016;28:8-13. doi:10.1016/j. coph.2016.02.009

2. Schaible HG. Mechanisms of chronic pain in osteoarthritis. Curr Rheumatol Rep. 2012;14(6):549-556. doi:10.1007/s11926-012-0279-x

3. Woolf CJ. Central sensitization: uncovering the relation between pain and plasticity. Anesthesiology. 2007;106(4):864-867. doi:10.1097/01. anes. 0000264769.87038 .55

4. Hochberg MC, Altman RD, April KT, et al. American College of Rheumatology 2012 recommendations for the use of nonpharmacologic and pharmacologic therapies in osteoarthritis of the hand, hip, and knee. Arthritis Care Res. 2012;64(4):465-474. doi:10.1002/acr.21596

5. Tavares D, Okazaki J, Rocha AP, et al. Effects of transcranial direct current stimulation on knee osteoarthritis pain in elderly subjects with defective endogenous pain-inhibitory systems: protocol for a randomized controlled trial. JMIR Res Protoc. 2018;7(10):e11660. doi:10.2196/11660

6. Hawker GA. Who, when, and why total joint replacement surgery? The patient's perspective. Curr Opin Rheumatol. 2006;18(5):526-530. doi:10.1097/01.bor.0000240367.62583.51

7. Bourne RB, Chesworth BM, Davis AM, Mahomed NN, Charron KD. Patient satisfaction after total knee arthroplasty: who is satisfied and who is not? Clin Orthop Relat Res. 2010;468(1):57-63. doi:10.1007/s11999-009-1119-9

8. Kwon M, Altin M, Duenas H, Alev L. The role of descending inhibitory pathways on chronic pain modulation and clinical implications. Pain Pract. 2014;14(7):656-667. doi:10.1111/papr.12145

9. Finnerup NB, Sindrup SH, Jensen TS. Chronic neuropathic pain: mechanisms, drug targets and measurement. Fundam Clin Pharmacol. 2007;21 (2):129-136. doi:10.1111/j.1472-8206.2007.00474.x

10. Staud R. Evidence for shared pain mechanisms in osteoarthritis, low back pain, and fibromyalgia. Curr Rheumatol Rep. 2011;13(6):513-520. doi:10.1007/s11926-011-0206-6

11. Porreca F, Ossipov MH, Gebhart GF. Chronic pain and medullary descending facilitation. Trends Neurosci. 2002;25(6):319-325. doi:10.1016/ S0166-2236(02)02157-4

12. Fields HL, Malick A, Burstein R. Dorsal horn projection targets of ON and OFF cells in the rostral ventromedial medulla. J Neurophysiol. 1995;74 (4):1742-1759. doi:10.1152/jn.1995.74.4.1742

13. Mantyh PW. Connections of midbrain periaqueductal gray in the monkey. II. Descending efferent projections. J Neurophysiol. 1983;49(3):582-594. doi:10.1152/jn.1983.49.3.582

14. Beitz AJ. The sites of origin brain stem neurotensin and serotonin projections to the rodent nucleus raphe magnus. $J$ Neurosci. 1982;2(7):829-842. doi:10.1523/JNEUROSCI.02-07-00829.1982

15. Jensen TS, Yaksh TL. The antinociceptive activity of excitatory amino acids in the rat brainstem: an anatomical and pharmacological analysis. Brain Res. 1992;569(2):255-267. doi:10.1016/0006-8993(92)90637-O

16. Davies SN, Lodge D. Evidence for involvement of N-methylaspartate receptors in 'wind-up' of class 2 neurones in the dorsal horn of the rat. Brain Res. 1987;424(2):402-406. doi:10.1016/0006-8993(87)91487-9

17. Dickenson AH, Sullivan AF. Evidence for a role of the NMDA receptor in the frequency dependent potentiation of deep rat dorsal horn nociceptive neurones following C fibre stimulation. Neuropharmacology. 1987;26(8):1235-1238. doi:10.1016/0028-3908(87)90275-9

18. Hu J, Wang Z, Guo YY, et al. A role of periaqueductal grey NR2B-containing NMDA receptor in mediating persistent inflammatory pain. Mol Pain. 2009;5:71. doi:10.1186/1744-8069-5-71

19. Zhuo M. Glutamate receptors and persistent pain: targeting forebrain NR2B subunits. Drug Discov Today. 2002;7(4):259-267. doi:10.1016/S13596446(01)02138-9

20. Gogas KR. Glutamate-based therapeutic approaches: NR2B receptor antagonists. Curr Opin Pharmacol. 2006;6(1):68-74. doi:10.1016/j. coph.2005.11.001

21. Nakazato E, Kato A, Watanabe S. Brain but not spinal NR2B receptor is responsible for the anti-allodynic effect of an NR2B subunit-selective antagonist CP-101,606 in a rat chronic constriction injury model. Pharmacology. 2005;73(1):8-14. doi:10.1159/000081069

22. Harris JA. Using c-fos as a neural marker of pain. Brain Res Bull. 1998;45(1):1-8. doi:10.1016/S0361-9230(97)00277-3

23. Coggeshall RE. Fos, nociception and the dorsal horn. Prog Neurobiol. 2005;77(5):299-352. doi:10.1016/j.pneurobio.2005.11.002

24. Miranda PC, Lomarev M, Hallett M. Modeling the current distribution during transcranial direct current stimulation. Clin Neurophysiol. 2006;117 (7):1623-1629. doi:10.1016/j.clinph.2006.04.009

25. Wagner T, Valero-Cabre A, Pascual-Leone A. Noninvasive human brain stimulation. Annu Rev Biomed Eng. 2007;9(1):527-565. doi:10.1146/ annurev.bioeng.9.061206.133100

26. Nitsche MA, Paulus W. Excitability changes induced in the human motor cortex by weak transcranial direct current stimulation. $J$ Physiol. 2000;527(Pt 3):633-639. doi:10.1111/j.1469-7793.2000.t01-1-00633.x

27. Nitsche MA, Fricke K, Henschke U, et al. Pharmacological modulation of cortical excitability shifts induced by transcranial direct current stimulation in humans. $J$ Physiol. 2003;553(Pt 1):293-301. doi:10.1113/jphysiol.2003.049916

28. Artola A, Brocher S, Singer W. Different voltage-dependent thresholds for inducing long-term depression and long-term potentiation in slices of rat visual cortex. Nature. 1990;347(6288):69-72. doi:10.1038/347069a0

29. Liebetanz D, Nitsche MA, Tergau F, Paulus W. Pharmacological approach to the mechanisms of transcranial DC-stimulation-induced after-effects of human motor cortex excitability. Brain. 2002;125(Pt 10):2238-2247. doi:10.1093/brain/awf238

30. Laste G, Caumo W, Adachi LN, et al. After-effects of consecutive sessions of transcranial direct current stimulation (tDCS) in a rat model of chronic inflammation. Exp Brain Res. 2012;221(1):75-83. doi:10.1007/s00221-012-3149-x

31. Roizenblatt S, Fregni F, Gimenez R, et al. Site-specific effects of transcranial direct current stimulation on sleep and pain in fibromyalgia: a randomized, sham-controlled study. Pain Pract. 2007;7(4):297-306. doi:10.1111/j.1533-2500.2007.00152.x

32. Fregni F, Boggio PS, Lima MC, et al. A sham-controlled, Phase II trial of transcranial direct current stimulation for the treatment of central pain in traumatic spinal cord injury. Pain. 2006;122(1-2):197-209. doi:10.1016/j.pain.2006.02.023

33. Ahn H, Woods AJ, Kunik ME, et al. Efficacy of transcranial direct current stimulation over primary motor cortex (anode) and contralateral supraorbital area (cathode) on clinical pain severity and mobility performance in persons with knee osteoarthritis: an experimenter- and participant-blinded, randomized, sham-controlled pilot clinical study. Brain Stimul. 2017;10(5):902-909. doi:10.1016/j.brs.2017.05.007 
34. Sajadi S, Karimi M, Forogh B, Raissi GR, Zarnegar F, Ahadi T. Randomized clinical trial comparing of transcranial direct current stimulation (tDCS) and transcutaneous electrical nerve stimulation (TENS) in knee osteoarthritis. Neurophysiol Clin. 2020;50(5):367-374. doi:10.1016/j. neucli.2020.08.005

35. Zimmermann M. Ethical guidelines for investigations of experimental pain in conscious animals. Pain. 1983;16(2):109-110. doi:10.1016/03043959(83)90201-4

36. Havelin J, Imbert I, Cormier J, Allen J, Porreca F, King T. Central sensitization and neuropathic features of ongoing pain in a rat model of advanced osteoarthritis. J Pain. 2016;17(3):374-382. doi:10.1016/j.jpain.2015.12.001

37. Watson C, Paxinos G. The Rat Brain in Stereotaxic Coordinates. 6th ed. Amsterdam: Academic Press/Elsevier; 2007.

38. Spezia AL, Quevedo AS, de Souza A, et al. Exogenously induced brain activation regulates neuronal activity by top-down modulation: conceptualized model for electrical brain stimulation. Exp Brain Res. 2015;233(5):1377-1389. doi:10.1007/s00221-015-4212-1

39. Leffa DT, Bellaver B, Salvi AA, et al. Transcranial direct current stimulation improves long-term memory deficits in an animal model of attention-deficit/hyperactivity disorder and modulates oxidative and inflammatory parameters. Brain Stimul. 2018;11(4):743-751. doi:10.1016/j. brs.2018.04.001

40. Lopes BC, Medeiros LF, Silva DSV, et al. Transcranial direct current stimulation combined with exercise modulates the inflammatory profile and hyperalgesic response in rats subjected to a neuropathic pain model: long-term effects. Brain Stimul. 2020;13(3):774-782. doi:10.1016/j. brs.2020.02.025

41. Chaplan SR, Bach FW, Pogrel JW, Chung JM, Yaksh TL. Quantitative assessment of tactile allodynia in the rat paw. J Neurosci Methods. 1994;53 (1):55-63. doi:10.1016/0165-0270(94)90144-9

42. Netto CA, Siegfried B, Izquierdo I. Analgesia induced by exposure to a novel environment in rats: effect of concurrent and post-training stressful stimulation. Behav Neural Biol. 1987;48(2):304-309. doi:10.1016/S0163-1047(87)90850-8

43. Hargreaves K, Dubner R, Brown F, Flores C, Joris J. A new and sensitive method for measuring thermal nociception in cutaneous hyperalgesia. Pain. 1988;32(1):77-88. doi:10.1016/0304-3959(88)90026-7

44. Okun A, Liu P, Davis P, et al. Afferent drive elicits ongoing pain in a model of advanced osteoarthritis. Pain. 2012;153(4):924-933. doi:10.1016/j. pain.2012.01.022

45. Millan MJ. The induction of pain: an integrative review. Prog Neurobiol. 1999;57(1):1-164. doi:10.1016/s0301-0082(98)00048-3

46. Reidler JS, Mendonca ME, Santana MB, et al. Effects of motor cortex modulation and descending inhibitory systems on pain thresholds in healthy subjects. J Pain. 2012;13(5):450-458. doi:10.1016/j.jpain.2012.01.005

47. Drouot X, Nguyen JP, Peschanski M, Lefaucheur JP. The antalgic efficacy of chronic motor cortex stimulation is related to sensory changes in the painful zone. Brain. 2002;125(Pt 7):1660-1664. doi:10.1093/brain/awf161

48. Lefaucheur JP. The use of repetitive transcranial magnetic stimulation (rTMS) in chronic neuropathic pain. Neurophysiol Clin. 2006;36(3):117-124. doi:10.1016/j.neucli.2006.08.002

49. Fregni F, Pascual-Leone A, Freedman SD. Pain in chronic pancreatitis: a salutogenic mechanism or a maladaptive brain response? Pancreatology. 2007;7(5-6):411-422. doi:10.1159/000108958

50. Nguyen JP, Nizard J, Keravel Y, Lefaucheur JP. Invasive brain stimulation for the treatment of neuropathic pain. Nat Rev Neurol. 2011;7 (12):699-709. doi:10.1038/nrneurol.2011.138

51. Lima MC, Fregni F. Motor cortex stimulation for chronic pain: systematic review and meta-analysis of the literature. Neurology. 2008;70 (24):2329-2337. doi:10.1212/01.wnl.0000314649.38527.93

52. Bandler R, Shipley MT. Columnar organization in the midbrain periaqueductal gray: modules for emotional expression? Trends Neurosci. 1994;17 (9):379-389. doi:10.1016/0166-2236(94)90047-7

53. Noh A, Ismail C. A review on chronic pain in rheumatoid arthritis: a focus on activation of NR2B subunit of N-Methyl-D-Aspartate receptors Malays J Med Sci. 2020;27(1):6-21. doi:10.21315/mjms2020.27.1.2

54. Sandkuhler J, Fu QG, Zimmermann M. Spinal pathways mediating tonic or stimulation-produced descending inhibition from the periaqueductal gray or nucleus raphe magnus are separate in the cat. J Neurophysiol. 1987;58(2):327-341. doi:10.1152/jn.1987.58.2.327

55. Paxinos G. The Rat Nervous System. 2nd ed. San Diego: Academic Press; 1995.

56. Wu LJ, Zhuo M. Targeting the NMDA receptor subunit NR2B for the treatment of neuropathic pain. Neurotherapeutics. 2009;6(4):693-702. doi:10.1016/j.nurt.2009.07.008

57. Ramos CM, Da SL, Menescal-de-oliveira L. Modulation of tonic immobility in Guinea pig PAG by homocysteic acid, a glutamate agonist. Physiol Behav. 2008;94(3):468-473. doi:10.1016/j.physbeh.2008.02.013

58. Berrino L, Oliva P, Rossi F, Palazzo E, Nobili B, Maione S. Interaction between metabotropic and NMDA glutamate receptors in the periaqueductal grey pain modulatory system. Naunyn Schmiedebergs Arch Pharmacol. 2001;364(5):437-443. doi:10.1007/s002100100477

59. Palazzo E, Marabese I, de Novellis V, et al. Metabotropic and NMDA glutamate receptors participate in the cannabinoid-induced antinociception. Neuropharmacology. 2001;40(3):319-326. doi:10.1016/S0028-3908(00)00160-X

60. Narita M, Ozaki S, Narita M, Ise Y, Yajima Y, Suzuki T. Change in the expression of c-fos in the rat brain following sciatic nerve ligation. Neurosci Lett. 2003;352(3):231-233. doi:10.1016/j.neulet.2003.08.052

61. Takeda R, Watanabe Y, Ikeda T, et al. Analgesic effect of milnacipran is associated with c-Fos expression in the anterior cingulate cortex in the rat neuropathic pain model. Neurosci Res. 2009;64(4):380-384. doi:10.1016/j.neures.2009.04.010

62. Fertonani A, Rosini S, Cotelli M, Rossini PM, Miniussi C. Naming facilitation induced by transcranial direct current stimulation. Behav Brain Res. 2010;208(2):311-318. doi:10.1016/j.bbr.2009.10.030

63. Krause B, Marquez-Ruiz J, Cohen KR. The effect of transcranial direct current stimulation: a role for cortical excitation/inhibition balance? Front Hum Neurosci. 2013;7:602. doi:10.3389/fnhum.2013.00602

64. Ngernyam N, Jensen MP, Auvichayapat N, Punjaruk W, Auvichayapat P. Transcranial direct current stimulation in neuropathic pain. J Pain Relief. 2013;Suppl 3. doi:10.4172/2167-0846.S3-001 


\section{Publish your work in this journal}

The Journal of Pain Research is an international, peer reviewed, open access, online journal that welcomes laboratory and clinical findings in the fields of pain research and the prevention and management of pain. Original research, reviews, symposium reports, hypothesis formation and commentaries are all considered for publication. The manuscript management system is completely online and includes a very quick and fair peer-review system, which is all easy to use. Visit http://www.dovepress.com/testimonials.php to read real quotes from published authors.

Submit your manuscript here: https://www.dovepress.com/journal-of-pain-research-journal 